\title{
Relationship between attack and pause in world taekwondo championship contests: effects of gender and weight category
}

\author{
Victor Gustavo Ferreira Santos ${ }^{1}$ \\ Flavio de Oliveira Pires ${ }^{2}$ \\ Romulo Bertuzzi ${ }^{3}$ \\ Emerson Frachini ${ }^{4}$ \\ Marcos David da Silva-Cavalcante ${ }^{1,3}$ \\ Maria Augusta Peduti Dal Molin Kiss ${ }^{3}$ \\ Adriano Eduardo Lima-Silva ${ }^{1}$ \\ 1 Department of Physical Education and Sports Science \\ (CAV), Federal University of Pernambuco, Brazil \\ 2 Catholic University of Brasilia, Brazil \\ ${ }^{3}$ Endurance Performance Research Group, School \\ of Physical Education and Sport, University of São \\ Paulo, Brazil \\ 4 Martial Arts and Combat Sports Research Group, \\ School of Physical Education and Sport, University \\ of São Paulo, Brazil
}

Corresponding author:

Adriano Eduardo Lima-Silva

Department of Physical Education and Sport Science (CAV), Federal University of Pernambuco

Alto do Reservatorio street, Bela vista

55608680 Vitoria de Santo Antáo, Pernambuco, Brazil

E-mail: adrianosilva@usp.br

\section{Summary}

Background: the aim of this study was to compare between weight and gender categories the attack and pause times during the 2007 Taekwondo World Championship. Methods: a total of 88 rounds (47 male and 41 female contests) were analyzed. $\boldsymbol{R e}$ sults: there was no difference in attack/balancing times ratio between genders $(0.13 \pm 0.06$ vs $0.13 \pm$ $0.06, P>0.05)$. The attack number was significantly higher in round 3 than in round 1 for all categories and genders. The balancing time was lower during the round 3 than round 1 for low-weight male and high-weight female categories. The delta of change from round 1 to 3 (round 3 - round 1) for attack time and attack/balancing times ratio were lower in male than female. Conclusions: female seems to intensify more the combat in the last round than male, and this seems be related to the weight division.

KEY WORDS: athletic performance, martial arts, sport, training, time and motion studies.

\section{Introduction}

The contests of World Taekwondo Federation (WTF) are characterized by fast kicks and punches interspersed by balancing periods used to prepare a new attack to the opponent ${ }^{1-4}$. The relationship between attack (AT) and balancing time (BT) during Taekwondo contests has been used to characterize the physical demand imposed by the task ${ }^{4}$. For example, a contest with long AT and short BT is considered more intense than a contest with short AT and long BT. In this respect, a complete understanding about AT and BT relationship would be useful to training prescription and adequate preparation for WTF's competitions ${ }^{4,5-9}$.

Previous studies investigating the relationship between AT and BT during taekwondo combats have frequently used time motion analyses because this method has been considered a valid and reliable tool ${ }^{4,5,8}$. A previous study from our group has confirmed that time motion analysis is highly reliable to measure AT, BT, AT/BT ratio, and attack numbers (AN) during WTF's combats ${ }^{4}$. Although time motion analysis does not allow a direct access of each energy system contribution during the combats, it could be an important tool to assist athletes and coaches when selecting an effort/pause ratio for a training session.

On the other hand, while combat details have been investigated in male competition ${ }^{4}$, females have not received the same attention. It may be important to investigate gender differences to develop specifics training program and combat strategies. In fact, one study has suggested that female winners used more techniques (defensive kicks, offensive kicks, and offensive/defensive punches) during the combat than their counterpart's male winners ${ }^{10}$. Therefore, an increased number of techniques expended in each combat may be more important for females than for males. However, it is not possible to identify from these findings whether the time expended during attacks, pauses and AT/BT ratio would be or not different between the genders. Thus, given the limited information available concerning the differences between genders during a Taekwondo combat, it is not possible at the moment to develop a gender-specific conditioning program based on the AT and BT relationship. A more personalized training program would be useful to avoid injury during the training sessions and increase training adaptations.

Therefore, the purpose of this study was to compare the AT, AN, BT and AT/BT ratio during the 2007 WTF's World Championship between weight cate- 
gories and genders. We hypothesized that time-motion analysis parameters derived from WTF's combats would be different between males and females. Specifically, we hypothesized that females would have a higher number attacks and a lower pause periods compared to males.

\section{Materials and methods}

\section{Video Analyses}

It was assessed a total of 88 rounds during the 2007 World Championship, in which 47 were male contests and 41 female contests. In order to reduce the influence of the combat performance level, we included in the analysis only decisive combats (semifinal and final). In order to generate a clear distinction between weight groups (lights vs. heavies) and a sufficient sample size, the lowest weight categories (Fin, Fly and Bantam) were grouped in a single category (low weight, LW), while the highest weight categories (Welter, Middle and Heavy) were grouped in another one (heavy weight, HW). The midway weight categories (Feather and Light) were excluded from analysis. This procedure guaranteed that LW and HW groups would be different in body mass ${ }^{11}$. Table 1 presents details about the combats assessed.

The design, implementation, evaluation, interpretation and report of the present study adhered to the standards and laws highlighted in the previous official editorial of MLTJ ${ }^{12}$.

\section{Procedure}

The official videos were obtained from official tapes recorded in DVD. All DVD videos were recorded by WTF. The videos were analyzed using the Nero Show Time program (Version 2, Toshiba Samsung Storage Technology Corporation, and Tokyo, Japan). The match comprised 3 rounds of 2 minutes each with 1 minute rest between them. The fourth round was not considered because it did not occur in all the matches (only in four male's matches). A single researcher highly experienced and familiarized with taekwondo matches analyzed all the videos in order to obtain the AT, $A N, B T$ and $A T / B T$ ratio for each round. This analysis followed the same procedures previously used in other studies, which shown to be highly reliable $4,9,13,14$.
The AT, AN, BT and AT/BT ratio were registered separately in each round. It was considered an attack when one of the athletes attacked or tried to attack the opponent. The time elapsed between the athlete began to move his foot or hand in direction to the opponent and the athlete finished attacking moves was considered as AT. The AN was considered the number of times that athletes attack the opponent. The BT was considered the total time in which there was no attempt to attack and time-out made by the referees. The AT/BT ratio was calculated dividing AT by BT. Deltas of change from round 1 to 3 were calculated to provide an estimate of the alterations crossing the combat.

\section{Statistical analysis}

The sample size was estimated using data from our previous study ${ }^{4}$. The data distribution was verified using Kolmogorov-Smirnov test. As non-normal distributions were found, a Mann-Whitney $U$ test was used to verify the significance of difference in AT, AN, BT and $A T / B T$ ratio indexes between genders. The indexes were also compared among weight divisions using Kruskal-Wallis followed by Mann-Whitney U test separately for each gender. The differences among rounds were also determined using the Friedman and Wilcoxon tests. The level of significance was set at $p<0.05$.

\section{Results}

The indexes for males and females are displayed in Table 2. Neither AT or BT were significantly different between male and female groups $(P>0.05)$. As a consequence, the AT/BT ratio was not significantly different between male and female $(P>0.05)$. The AN was also similar between genders $(P>0.05)$.

The round indexes for male and female are displayed in Table 3. When the rounds were averaged (average per combat), results showed no differences between weight or genders categories $(P>0.05)$. However, the indexes changed consistently from round 1 to round 3 , and these changes were gender and weight dependents $(P<0.05)$. The $A N$ in the LW category for male was significantly higher and BT was significantly lower during round 3 than rounds 1 and $2(\mathrm{P}<$ $0.05)$. For $\mathrm{HW}$ category, only the AN was significantly higher in round 3 than round $1(P<0.05)$. On the other hand, the AN was significantly higher during round

Table 1. Characteristics of the contests analysed during 2007 World Championship.

\begin{tabular}{lllll}
\hline & \multicolumn{2}{c}{ Female } & \multicolumn{2}{c}{ Male } \\
\hline Weight & Round Investigated & Weight & Round Investigated \\
\hline HW & $\leq 55 \mathrm{~kg}$ & 9 semifinals and 9 finals & $\leq 62 \mathrm{~kg}$ & 15 semifinals and 9 finals \\
\hline
\end{tabular}

LW: Pooled light categories (Fin, Fly and Bantam); HW: Pooled heavy categories (Welter, Middle and Heavy). 
3 compared to round 1 for female in the LW category $(P<0.05)$. For HW category in female, the $A N$ and AT/BT ratio were higher in round 3 than rounds 1 and 2 , while the BT was lower in round 3 than in round 1 $(\mathrm{P}<0.05)$.

When the magnitude of changes between round 1 and 3 were compared between genders (delta values), $\triangle \mathrm{AT}$ and $\triangle \mathrm{AT} / \mathrm{BT}$ ratio were lower in male than female $(P<0.05$, Table 4). However, when the analysis was separated by weight division, only the $\Delta \mathrm{AT}$ was significantly lower in the HW male than in the $\mathrm{HW}$ female $(\mathrm{P}<0.05)$. There was also a difference in the $\triangle \mathrm{AN}$ between the weight divisions for female ( $\mathrm{LW}$ vs. HW female, $\mathrm{P}<0.05)$.

\section{Discussion}

The purpose of this study was to determine the AT, AN, BT and AT/BT ratio during the 2007 WTF's World

Table 2. Values (mean \pm SD) for attack time (AT), balancing time (BT), attack number (AN), AT/BT ratio for male and female groups during the combats of the 2007 World Championship.

\begin{tabular}{lllll}
\hline & $\begin{array}{l}\text { Male } \\
(\mathbf{n = 4 7 )}\end{array}$ & $\begin{array}{l}\text { Female } \\
(\mathbf{n}=\mathbf{4 1 )}\end{array}$ & $\begin{array}{l}\text { Pooled group } \\
(\mathbf{n = 8 8})\end{array}$ & P value \\
\hline AT (s) & $1.5 \pm 0.3$ & $1.5 \pm 0.3$ & $1.5 \pm 0.3$ & 0.400 \\
AN (times) & $9.8 \pm 4.0$ & $9.8 \pm 3.6$ & $9.8 \pm 3.8$ & 0.966 \\
BT (s) & $14.4 \pm 5.8$ & $13.8 \pm 6.5$ & $14.1 \pm 6.1$ & 0.459 \\
AT/ BT Ratio & $0.13 \pm 0.06$ & $0.13 \pm 0.06$ & $0.13 \pm 0.06$ & 0.695 \\
\hline
\end{tabular}

Table 3. Values (mean \pm SD) for attack time (AT), balancing time (BT), attack number AN, AT/BT ratio in rounds one, two and three of the combats of the 2007 World Championship.

\begin{tabular}{|c|c|c|c|c|c|c|c|c|}
\hline & \multicolumn{4}{|c|}{ LW male $(n=24)$} & \multicolumn{4}{|c|}{ LW female $(n=18)$} \\
\hline & Round 1 & Round 2 & Round 3 & Average & Round 1 & Round 2 & Round 3 & Average \\
\hline AT (s) & $1.5 \pm 0.4$ & $1.6 \pm 0.5$ & $1.5 \pm 0.3$ & $1.5 \pm 0.4$ & $1.4 \pm 0.2$ & $1.5 \pm 0.2$ & $1.9 \pm 0.5$ & $1.6 \pm 0.3$ \\
\hline AN (times) & $9.1 \pm 2.3^{*}$ & $8.4 \pm 2.9^{\star}$ & $13.8 \pm 3.9$ & $10.4 \pm 3.8$ & $9.2 \pm 1.9^{*}$ & $9.7 \pm 2.3$ & $11.6 \pm 2.5$ & $10.1 \pm 2.4$ \\
\hline $\mathrm{BT}(\mathrm{s})$ & $13.6 \pm 3$ & $15.2 \pm 5.8^{*}$ & $10.4 \pm 3.1$ & $13.1 \pm 4.7$ & $13.8 \pm 3.5$ & $11.4 \pm 3.9$ & $10.9 \pm 0.9$ & $12.1 \pm 3.3$ \\
\hline \multirow[t]{3}{*}{ AT/BT ratio } & $0.14 \pm 0.05$ & $0.13 \pm 0.07$ & $0.15 \pm 0.08$ & $0.14 \pm 0.06$ & $0.12 \pm 0.04$ & $0.13 \pm 0.05$ & $0.18 \pm 0.04$ & $0.14 \pm 0.05$ \\
\hline & \multicolumn{4}{|c|}{ HW male $(n=23)$} & \multicolumn{4}{|c|}{ HW female $(n=23)$} \\
\hline & Round 1 & Round 2 & Round 3 & Average & Round 1 & Round 2 & Round 3 & Average \\
\hline AT (s) & $1.5 \pm 0.3$ & $1.4 \pm 0.2$ & $1.4 \pm 0.2$ & $1.4 \pm 0.2$ & $1.4 \pm 0.3$ & $1.5 \pm 0.2$ & $1.7 \pm 0.4$ & $1.5 \pm 0.3$ \\
\hline AN (times) & $7.1 \pm 3.8^{\star}$ & $8.5 \pm 3.1$ & $12.4 \pm 4.3$ & $9.2 \pm 4.2$ & $7.0 \pm 2.8^{*}$ & $7.9 \pm 2.4^{*}$ & $14.3 \pm 3.5$ & $9.5 \pm 4.3$ \\
\hline $\mathrm{BT}(\mathrm{s})$ & $18.9 \pm 8.7$ & $15.7 \pm 4.6$ & $12.5 \pm 4.2$ & $15.8 \pm 6.6$ & $19.6 \pm 10.5^{\star}$ & $14.6 \pm 5.3$ & $10.3 \pm 4.1$ & $15.0 \pm 7.9$ \\
\hline AT/BT ratio & $0.11 \pm 0.06$ & $0.10 \pm 0.00$ & $0.13 \pm 0.05$ & $0.11 \pm 0.05$ & $0.09 \pm 0.04^{*}$ & $0.10 \pm 0.05^{*}$ & $0.19 \pm 0.07$ & $0.12 \pm 0.07$ \\
\hline
\end{tabular}

* Significantly different from round $3(\mathrm{P}<0.05)$. HW = heavy weight, $\mathrm{LW}=$ low weight.

Table 4. Deltas calculated from the difference between the round 1 and round 3.

\begin{tabular}{lll}
\hline & Male (pooled data, $\mathbf{n}=\mathbf{4 7})$ & Female $($ pooled data, $\mathbf{n}=\mathbf{4 1})$ \\
\hline$\Delta$ AT & $-0.06 \pm 0.31^{*}$ & $0.33 \pm 0.37$ \\
$\Delta$ AN & $4.80 \pm 3.80$ & $5.17 \pm 3.74$ \\
$\Delta$ BT & $-4.93 \pm 7.06$ & $-6.34 \pm 10.16$ \\
$\Delta$ AT/BT ratio & $0.01 \pm 0.06^{*}$ & $0.08 \pm 0.08$ \\
\hline & LW Male $(\mathbf{n}=\mathbf{2 4})$ & LW Female $(\mathbf{n}=\mathbf{1 8})$ \\
\hline$\Delta$ AT & $-0.05 \pm 0.35$ & $0.42 \pm 0.49$ \\
$\Delta$ AN & $4.63 \pm 3.42$ & $2.00 \pm 1.58 \dagger$ \\
$\Delta$ BT & $-3.14 \pm 3.64$ & $-2.04 \pm 2.46$ \\
$\Delta$ AT/BT ratio & $0.01 \pm 0.06$ & $0.06 \pm 0.05$ \\
\hline & HW Male $(\mathbf{n}=\mathbf{2 3})$ & HW Female $(\mathbf{n}=\mathbf{2 3})$ \\
\hline$\Delta$ AT & $-0.07 \pm 0.27 *$ & $0.27 \pm 0.29$ \\
$\Delta$ AN & $5.00 \pm 4.47$ & $7.43 \pm 3.10$ \\
$\Delta$ BT & $-6.96 \pm 9.58$ & $-9.41 \pm 12.60$ \\
$\Delta$ AT/BT ratio & $0.01 \pm 0.07$ & $0.10 \pm 0.10$
\end{tabular}

* Significantly different from Female in the same weight category. † Significantly different from HW female. 
Championship combats and to compare the values among the rounds, genders and weight divisions. We found that average AT, AN, BT and AT/BT ratio were not different between male and female combats. However, females intensified more the combat in the last round when compared with the first round. This last one was more evident for HW than for LW division.

In the present study, it was found an AT/BT ratio of $0.13 \pm 0.06$ for both genders. Matsughigue et al. ${ }^{8}$ analyzed contests of Songham Taekwondo and found an AT/BT ratio of $\sim 0.16$ when considering high intensity effort and pause ratio. Similarly, Santos et al. ${ }^{4}$ analyzed male combats of 2007 World Taekwondo Championship and 2008 Olympics Games and found a ratio between attack time and skipping time from $\sim 0.18 \pm 0.12$. Similar results were reported by Campos et al. ${ }^{9}$ and Heller et al. ${ }^{5}$ analyzing ITF Taekwondo contests in both genders. Together, these results indicate that during taekwondo contests the time expended in high intensity effort is much lower than in low intensity effort.

The AN observed in the present study was $9.8 \pm 3.8$ attacks per round, similar to those found in previous studies $^{4,15}$, but is lightly different from others ${ }^{6,9}$. Butios and Tasika ${ }^{6}$ have showed that during simulations of WTF contest the attack number ranged from 15 to 20 attacks per round. Campos et al. ${ }^{9}$ have found that athletes performed $18 \pm 3$ attacks during a combat simulation. These differences could be credited mainly to time into each round ( 3 versus 2 minutes), and type of combat (simulations versus real competition), which would result in technical and tactical differences.

The results of the present study indicated that the third round is most intense than the first round. The AN increased and BT reduced during the round 3 in all weight divisions and genders. It is interesting to observe that BT was reduced in round 3 only for $\mathrm{LW}$ male and HW female divisions. The reduction in BT during the round 3 was lesser pronounced in LW female and HW male divisions. These results suggest that athletes adopted a progressive fight pacing strategy, increasing progressively the number of attacks from first to last round. The intensification of the combat during the last round may be explained by athletes whom were losing may have tried to attack more times and score more points in order to revert the combat, while the athletes whom were winning may have been obligated to counterattack, increasing therefore the intensity of the combat ${ }^{16}$. Additionally, athletes may spend more time in the first round analyzing the opponent, and may prefer to decide the combat in the last round. It is noteworthy that we identified similar results for HW female and LW male. A possible explanation for this result is that the mean weight in these two categories were similar, i.e. $63 \mathrm{~kg}$ and $\sim 62 \mathrm{~kg}$, respectively, suggesting that decline in BT may be more dependent of the weight than of gender.

The results of the present study also showed that $\triangle \mathrm{AT} / \mathrm{BT}$ ratio was significantly higher for female than male, suggesting that female may have intensified more the combat from round 1 to round 3 . Kazemi et al. ${ }^{10}$ suggested that women winners use more tech- niques (defensive kicks, offensive kicks, and offensive/defensive punches) during the combat than their counterpart's male winners. Another possible explanation may be related to body mass because heavier athletes presented higher AT/BT ratio than lighter athletes. In fact, females are lighter than male athletes so females should have lower AT/BT ratio than males. It is interesting to note that $\triangle \mathrm{AT}$ was negative for male and positive for female, but it was evident only for HW division. This result suggests that male athletes are faster than females in round 3 and/or lesser fatigable. The present study presents some relevant strengths and weaknesses points. This is the first study showing that athletes adopted any type of "pacing strategy" during the combat, and it may be gender and weight dependent. However, this study presents some relevant limitations. It is important to note that time-motion analyses may involve subjective perceptions and decisions of the investigator. Although this technique has been demonstrated to be highly objective and reliable ${ }^{4}$, any error derived from video analyses cannot be fully disregarded. Nevertheless, these results might impact on clinical practice. Pacing strategies throughout the rounds should be taken into account when prescribing training for Taekwondo athletes and during official contests, considering weight and genders differences. This more individualized training prescription may reduce injury and increase training adaptations. Further studies should investigate these suppositions.

A summary of the main outcomes of the present study includes: 1) athletes adopted any type of "pacing strategy" from round 1 to round 3 , intensifying the combats in the last round; 2) females intensified more the combat than males from round 1 to round 3 and this seemed to be weight-dependent and; 3) the decline in balancing time in the last round seems be more dependent of weight than gender. Considering sports medicine issues and clinical practice, our findings suggest that: 1) pacing strategies throughout the three rounds should be taken into account when prescribing training for Taekwondo athletes; 2) coaches and trainers should consider differences of the combat's dynamics between weight and genders categories when prescribing training for Taekwondo athletes and; 3) coaches, trainers and/or athletes might elaborate specific combat strategies during official contests.

In conclusion, it was found in the present study that female intensified more the combat in the last round than male, and it was related to weight division. From a practical standpoint, results of the present study can aid coaches and trainers in training prescription of taekwondo preparation, in which weight category and gender should be taken into account. In addition, these results could support the elaboration of specific combat strategies for male and female categories during official contests.

\section{Acknowledgments}

We are grateful to Debora Brenner and Gabriela Nascimento for technical assistance. 


\section{Competing interests}

The authors declare no conflict of interest.

\section{Funding}

No financial support was received.

\section{Authors Contribution}

VGFS, AEL-S, RB and MDS-C were involved in the design of the experiment, collection of the data, data analysis and interpretation and writing of the manuscript. FOP, MAPDMK and EF were involved in the data acquisition and data analysis (especially the statistical analyses), and writing of the manuscript.

\section{References}

1. Pieter F, Pieter W. Speed and force in selected Taekwondo techniques. Biol Sport. 1995;12:257-266.

2. Kazemi M, Casella C, Perri G. 2004 Olympic Tae Kwon Do athlete profile. J Can Chiropr Assoc. 2009;53:144-152.

3. Kazemi M, Perri G, Soave D. A profile of 2008 Olympic Taekwondo competitors. J Can Chiropr Assoc. 2010;54:243-249.

4. Santos VGF, Franchini E, Lima-Silva AE. Relationship between attack and skipping in Taekwondo contests. J Strength Cond Res. 2011;25:1743-1751.

5. Heller J, Peric T, Dlouhá R, et al. Physiological profiles of male and female taekwon-do (ITF) black belts. J Sports Sci. 1998 16:243-249.

6. Butios S, Tasika N. Changes in heart rate and blood lactate concentration as intensity parameters during simulated Taekwondo competition. J Sports Med Phys Fitness. 2007;47:179-185.

7. Bridge CA, Jones MA, Drust B. Physiological Responses and Perceived Exertion During International Taekwondo Competition. Int J Sports Physiol Perform. 2009;4:485-493.

8. Matsushigue KA, Hartmann K, Franchini E. Taekwondo: Physiological responses and match analysis. J Strength Cond Res. 2009;23:1112-1117.

9. Campos FA, Bertuzzi R, Dourado AC, et al. Energy demands in Taekwondo athletes during combat simulation. Eur J Appl Physiol. 2012;112:1221-1228.

10. Kazemi M, Waalen J, Morgan C, White A. A profile of Olympic Taekwondo competitors. J Sports Sci Med. 2006; 5:114-121.

11. Cohen J, Cohen P. Applied multiple regression/correlation analysis for the behavioral sciences. Hillsdale; Erlbaum 1983.

12. Padulo J, Oliva F, Frizziero A, Maffulli N. Muscles, Ligaments and Tendons Journal. Basic principles and recommendations in clinical and field science research. MLTJ. 2013;4:250-252.

13. Salvador A, Martinez-Sanchis S, Simon VM, et al. Correlating testosterone and fighting in male participants in judo contests. Physiol Behav. 1999; 68:205-209.

14. Miarka B, Hayashida CR, Julio UF, et al. Objectivity of FRAMISoftware for Judo Match Analysis. Int J Perform Anal Sport. 2011;11:254-266.

15. Bouhlel E, Jouini A, Gmada N, et al. Heart rate and blood lactate responses during Taekwondo training and competition. Science \& Sports. 2006;21:285-290.

16. Chiodo $\mathrm{S}$, Tessitore $\mathrm{A}$, Cortis $\mathrm{C}$, et al. Effects of official taekwondo competitions on all-out performances of elite athletes. $J$ Strength Cond Res. 2011;25:334-339. 